\title{
Detection of Super-High-Frequency Partial Discharge by Using Neural Networks
}

\author{
Qingshan Shan, Shahzad Bhatti, I A Glover, R Atkinson, and R Rutherford
}

\begin{abstract}
- a system has been developed for the detection of super-high-frequency (SHF) partial discharge (PD) at frequencies up to $6 \mathrm{GHz}$. The system consists of 3 antennas for capturing PDs and a fast digital oscilloscope for sampling data. One antenna is a disk-cone antenna with frequency range below 710 MHz. The other two half TEM horn antennas have been designed and constructed for the frequency range 716 $\mathrm{MHz}$ - $5 \mathrm{GHz}$. To extend the frequency range up to $6 \mathrm{GHz}$ a methodology has been developed by compensating amplituderesponse to frequency-magnitude. The compensation is realized by using multilayer feed-forward neural networks to equalize on amplitude-response to frequency-magnitude. A direct sampling method is used to $\log$ the captured PD data. This PD detection system has been implemented to measure PDs at a 400 kV electrical substation (Strathaven, Scottish Power Ltd).
\end{abstract}

Index Terms - Partial discharge, TEM horn, Neural network, Super high frequency

\section{INTRODUCTION}

$\mathrm{T}$ HERE is a significant amount of instrumentation and control equipment located throughout electricity power substations for monitoring its state. Information and control signals for both normal and abnormal operation are traditionally connected, using cables or optical fibres, to a SCADA (Supervision, Control and Data Acquisition) system [1] and/or its successor UCA (Utilities Communication Architecture) systems [2]. Ethernet local area network (LAN) implementations of such UCA/SCADA systems, which simplify the addition/reconfiguration of instrumentation and the coordination of protection systems, have been proposed and are already being evaluated [3]. Significant flexibility and cost advantages over a wired LAN infrastructure could be gained, however, if signals were routed wirelessly. Furthermore, wireless communication technologies hold out the prospect of 'hot-line', sensors that can be deployed on

Manuscript received October 9, 2001. This work was supported by The UK Engineering \& Physical Sciences Research Council (EPSRC) under Grant EP/D049687/1.

Qingshan Shan, Shahzad Bhatti, I A Glover, and R Atkinson are with the Department of Electronic \& Electrical Engineering, University of Strathclyde, Glasgow, G1 1XW, UK (phone: +44-141-5482663; fax: +44-141-5524968; email: qingshan.shan@ eee.strath.ac.uk).

$R$ Rutherford is with Scottish Power - Energy, Networks \& Telecommunications, 1 Atlantic Quay, Glasgow, G2 8SP, UK (e-mail: Richard.Rutherford@sppowersystems.com).

XXX is with the Electrical Engineering Department, University of Colorado, Boulder, CO 80309 USA, on leave from the National Research Institute for Metals, Tsukuba, Japan (e-mail: author@nrim.go.jp).

*Reproduced from the authors existing paper [8] energized high-voltage (HV) equipment without the inconvenience and costs associated with bridging the system's primary insulation [4, 5]. Wireless LAN and Wireless Personal Area Network (PAN) technologies represent obvious opportunities to realize these advantages. Whilst the naturally occurring noise environment is relatively benign at Wireless LAN (2.4 and $5.5 \mathrm{GHz})$ and Wireless PAN (2.4 GHz) frequencies [6], the man-made noise environment within a substation is complex and hostile due, for example, to partial discharge (PD) from imperfect insulation and sferic radiation from switching and fault transients. The term sferic usually relates to radiation from a lightening event but is used here as a shorthand for similar radiation arising from any large current transient. An investigation into the vulnerability of WLAN and WPAN technologies to impulsive noise in electricity transmission substations has been proposed [7]. One of the major objectives of this investigation is the detection of PD and sferic radiation in electrical substations. In order to assess the impact of PD and sferic radiation on Wireless LAN (802.11 $\mathrm{a} / \mathrm{b} / \mathrm{g}$ ) and Wireless PAN technologies, the spectral characteristics of the PD up to $6 \mathrm{GHz}$ is of particular interest.

PD is an electrical discharge that fails to fully bridge the space between a pair of electrodes. It can occur around an electrode in a gas (corona), within gas bubbles in a liquid or within the space created by voids in a solid. HV plant (transformers, switchgear, cables etc) is especially prone to PD if its insulation is damaged and/or as its insulation ages. If remedial action is not taken the insulation can be seriously compromised leading, ultimately, to catastrophic failure. Energy from PD processes can be radiated whenever spectral components arising from current pulse edges extend into the radio frequency (RF) region [8]. Although the character of PD appears to have some dependence on the size and geometry of plant components (e.g. insulating spacers, Lshaped buses, T-branch buses) damping typically appears to become significant between $100 \mathrm{MHz}$ and $300 \mathrm{MHz}$ and increases with increasing frequency above this range [9]. PD energy in the frequency range $0.5-1.2 \mathrm{GHz}$, however, is readily radiated from apertures formed, for example, by insulating spacers or bushings [13]. PD current pulses in strong insulators (e.g. $\mathrm{SF}_{6}$ ) can have rise-times as short as 50 ps and may contain significant energy at frequencies up to 3 $\mathrm{GHz}[10]$.

Methods and systems for the detection of very-highfrequency (VHF) (up to $300 \mathrm{MHz}$ ) and ultra-high-frequency (UHF) (up to $3 \mathrm{GHz}$ ) PD in GIS have been developed [10- 
12]. These represent intrusive approaches to detect PD by using a VHF or UHF coupler which is mounted on GIS equipment. A non-intrusive PD measurement based on RF technology is capable of detecting frequencies up to $1.2 \mathrm{GHz}$ [13]. However, the authors are unaware of any investigation on detection of PD at frequencies up to $6 \mathrm{GHz}$ in a nonintrusive manner. This paper will present a SHF PD detection system which is capable of measuring up to $6 \mathrm{GHz}$ signals. The key issues addressed are the PD measurement system, the methodology of frequency bandwidth expanding, the neural network for equalization and the analysis of PD spectrum.

\section{Measurement System}

The site for PD detection was at Strathaven Substation and belongs to Scottish Power Ltd in UK, as shown in Fig. 1. The site is a combination of $400 \mathrm{kV}, 275 \mathrm{kV}$ and $132 \mathrm{kV}$ air insulation power substations. A PD detection system was deployed in the control building of the $400 \mathrm{kV}$ power substation, as shown in Fig. 2. The system consists of a low band (LB) TEM horn antenna, a high band (HB) TEM horn antenna, a disk-cone antenna, a WLAN dual band antenna, a digital storage oscilloscope (DSO) and an external hard drive disk (HDD). The schematic of the system is shown in Fig. 3. A detailed description of each antenna can be found in [13, 14]; however, a brief description will be provided in this paper for completeness.

The half TEM horn has been selected to detect PD due to its good impulse response. The schematic of the TEM horn architecture is shown in Figure 4. The LB TEM horn is constructed from a triangular aluminum plate and a $122 \mathrm{~cm} \times 122 \mathrm{~cm}$ aluminum ground plane [14]. The triangular plate's width $(w)$ at the aperture is $65.1 \mathrm{~cm}$, its length $(L)$ is $84 \mathrm{~cm}$ and its aperture height $(h)$ is $20.1 \mathrm{~cm}$. The antenna feed is a $50 \Omega$ SMA connector with its flange in electrical contact with the ground plane and its centre-conductor connected to the triangular plate apex. The $-3 \mathrm{~dB}$ bandwidth is $1.264 \mathrm{GHz}$ covering the frequency range $716 \mathrm{MHz}-1.98 \mathrm{GHz}$ by the measured amplitude response (frequency response magnitude) with a network analyzer, as shown in Fig. 6. The peak value occurs at $1.068 \mathrm{GHz}$.

The HB TEM horn is constructed using a PCB for the triangular flange and a $122 \mathrm{~cm} \times 122 \mathrm{~cm}$ aluminum plate for the ground plane [14]. The flange width $(\mathrm{w})$ at the aperture is $21.7 \mathrm{~cm}$ and its length $(\mathrm{L})$ is $28 \mathrm{~cm}$. The aperture height $(\mathrm{h})$ is $6.7 \mathrm{~cm}$. The feed structure and ground plane are identical to those of the LB horn. The amplitude response, measured with network analyzer, shows that the $-3 \mathrm{~dB}$ bandwidth to be $3.195 \mathrm{GHz}$ (pass-band 1.905 to $5.1 \mathrm{GHz}$ ) with a peak value at $2.13 \mathrm{GHz}$, as shown in Fig. 7.

The disk-cone antenna is only used to cover frequency range below $710 \mathrm{MHz}$ for PD detection due to its bandwidth characteristics. The disk-cone antenna consists of the cone section and the base (ground) plate [13]. The base plate is $171 \mathrm{~cm}$ diameter of aluminum plate. The cone section was machined from solid aluminum, $13.3 \mathrm{~cm}$ diameter of the joint section and $5.4 \mathrm{~cm}$ height of the lower part. The schematic of the disk-cone antenna is shown in Figure 5. It provides flat frequency response of the vertical electric field in the range $0-2.5 \mathrm{GHz}$ with a constant impedance $50 \Omega$.

To assess the response of the WLAN antenna to PD in the substation, a dual band WLAN antenna, which is commercial available and works at bands $2.4 \mathrm{GHz}$ and $5.5 \mathrm{GHz}$, was used to capture PD directly. The DSO is a LeCroy SDA 9000 with 4 simultaneous sampling channels. Each channel the sampling rate is $20 \mathrm{GS} / \mathrm{s}$, the RAM is $50 \mathrm{Mpts}$, the bandwidth is $6 \mathrm{GHz}$, and input impedance $50 \Omega$. The external HDD has 1000 GB memory space.

The antennas were directly connected to the DSO; direct sampling was used since previous studies [15] have shown receiver down-conversion distorts the signal. Due to the high electric field environment in which this system works, minimizing any possible coupling introduced through the connections between antennas and the DSO is vital. This was achieved via the use of $18 \mathrm{GHz} \& 50 \Omega$ coaxial cables. The conventional amplitude triggering of the DSO was used. The 4 channels simultaneously recorded signals at $20 \mathrm{GS} / \mathrm{s}$. Each signal length recorded is $2.5 \mathrm{~ms}$, which is the longest possible and limited by the channel RAM size. The recorded signals were saved to the external HDD, which was connected to the DSO through a USB interface. The cycle was repeated until the external HDD was filled.

\section{Methodology of Frequency Bandwidth ExPanding}

The LB and HB TEM horns, mentioned in previous section, have a combined bandwidth which is less than the 6 $\mathrm{GHz}$ required. There is approximately a $10 \mathrm{~dB}$ gain difference from LB to HB TEM horn which might affect the accuracy of PD spectrum analysis. To address this problem and improve the flatness of the amplitudes to the frequency magnitude for the TEM horns, a bandwidth expanding methodology is designed. The principle of the methodology is that a time-domain signal is transformed to a frequencydomain signal, then the frequency-domain signal is equalized and finally the equalized frequency-domain signal is transformed back to the time-domain signal. A block diagram of the methodology is shown in Fig. 8. The original timedomain signal is transformed to a frequency-domain representation using the Fast Fourier Transforms (FFT); the FFT output is expressed in magnitude and phase. The magnitudes are compensated with an equalizer which is controlled by the frequency and phase from the FFT outputs. The equalized magnitudes and the previous phase angles are converted to complex data. The complex data (frequencydomain) are transformed back to the time-domain signal using the inverse FFT. Finally, an equalized time-domain signal is obtained. The key part of this methodology is the equalizer which uses a trained Artificial Neural Network (ANN).

An ANN is capable of 'learning' by using experience to improve its performance [16]. When an ANN is exposed to a sufficient number of samples, it can generalize its response to 
to others it has not yet encountered. An ANN consists of a number of interconnected processors (neurons) which are analogous to the biological neurons in the brain. The neurons are connected by weighted links. The weights are the means of long-term memory in ANNs; they express the strength of each neuron input. An ANN 'learns' through repeated adjustments of these weights. In this study, multilayer feedforward neural networks (MFNN) are trained to be the equalizer. The MFNN, a type of $\mathrm{ANN}$, has been widely used in function approximation, pattern recognition and pattern classification. A MFNN consists of an input layer of source neurons, at least one hidden layer of computational neurons, and an output layer of computational neurons. One of most popular learning algorithms for MFNN is back-propagation. A set of training input patterns is presented to the network. The network computes its output pattern and if there is an error, the weights are adjusted to reduce this error. The input signals are propagated in a forward direction on a layer-bylayer basis. The error signal propagation starts at the output layer and work backwards to the hidden layers.

The frequency-band-expanding methodology is used to post-process PD data measured by the LB and HB TEM horns.

\section{NeURAL Network FOR EQUalization}

The equalizer uses the MFNN to map the time domain amplitude of the TEM horns' signals to the frequency domain magnitude and phase components. The inputs of MFNN are these frequency and phase components and the output is the compensated frequency domain magnitude. In order to have the same level of the amplitude response to frequency magnitude for the LB and HB TEM horns' signals, the target of the compensation of amplitude responses is referred to LB TEM horn's maximum amplitude value. This maximum value is subtracted from the rest of data of LB and HB TEM horns' amplitude responses. The results obtained from the subtraction are the compensation data for LB and HB TEM horn as shown in Fig. 12 and 13 and labeled 'Proposed', respectively. They are the target data for training MFNNs.

\section{A. Architecture}

The architecture of MFNN used in this study is shown in Fig. 9. The MFNN consists of an input layer, 2 hidden layers and an output layer. The first and second hidden layers consist of 3 neurons each. There are 2 types of transfer function, which are applied to each layer's output, and hence as an input to the next layer. A hyperbolic tangent sigmoid is used as an input to the first and second hidden layers and a linear function is used as an input to the output layer. The same architecture for the MFNN is used for both LB and HB TEM horns' equalizers.

\section{B. Learning and Training}

The gradient descent with momentum algorithm is used for the MFNN learning because this learning method can avoid local minima, speed up learning and stabilize convergence [17]. The learning is to calculate the weight change for a given neuron from the neuron's input and error, the weight (or bias), learning rate and momentum constant according to gradient descent with momentum. The Levenberg-Marquardt algorithm is used to train the neural network due to its efficiency [18]. Training is used to update the weights and bias their values according to Levenberg-Marquardt optimization. The mean squared error (MSE) is used to evaluate the network's performance. The method of training networks and the training data sets form for LB and HB TEM horns is identical. The following details the training the network for the LB TEM horn as an example.

There are 201 empirically derived input and target data sets available that can be used to train and validate the behavior of the neural network. The input data sets consist of frequency domain magnitude and phase values. The target data sets (i.e. expected outputs) are the magnitude values for compensation, as shown in Fig. 12 and labeled 'Proposed', corresponding to the frequency in the input data sets. The 201 data sets are divided to three subsets. The first subset with 151 data sets forms the training data sets. The second subset with 29 data sets forms the check data sets. The third subset with 21 data sets forms the test data sets. The training subset is used to compute and update the network weights and biases. The check subset is used to monitor the training procedure avoiding network overfitting. Overfitting arises when the error on the training set is driven to a very low value, but when new data is presented to the network the error is large. This can occur when the network has memorized the training example, yet has not learned to generalize new inputs. The check subset is a validation set; the validation error normally decreases during the initial stage of training, as does the training set error. When the network begins to overfit the data, the error on the validation set will typically begin to rise. When the validation error increase for a specified number of iterations, the training is stopped and the weights and biases at the minimum of the validation error are returned. The test subset is used to assess the trained network performance. The training goal is set to $10^{-5}$ (MSE). The maximum training epochs are set to 300 . The training terminates at $22^{\text {nd }}$ epoch. The performances for LB TEM horn's network during training are shown in Fig. 10. The $-O$ - line represents the training results. The $-\square$ - line represents the validation results. The -*- line represents the test results. Although the network training has terminated at 0.0035946 MSE instead of the setting goal, the accuracy of network model is deemed acceptable.

The training of HB TEM horn's network terminates at $42^{\text {th }}$ epoch. The performances of the network during training are shown in Fig. 11. The -O- line represents the training results. The $-\square$ - line represents the validation results. The -*- line represents the test results. The network training has terminated before reaches the setting goal. The training accuracy $0.00143883 \mathrm{MSE}$ is acceptable. 


\section{Performance}

The LB horn's frequency domain magnitude for the proposed compensation scheme and empirically derived values with MFNN are shown in Fig. 12. Similarly, those for the HB horn are shown in Fig 13. In both figures, the solid line represents the proposed equalization values while the solid *- line represents the empirical values.

Fig. 12 demonstrates that the LB TEM horn's frequency band has been expanded to $710 \mathrm{MHz}-2.1 \mathrm{GHz}$. Within this frequency band the magnitude flatness is less than $2.995 \mathrm{~dB}$ after the MFNN's equalization. Fig. 13 demonstrates that the frequency band of the HB TEM horn has been expanded to 2.1 - $6 \mathrm{GHz}$. Within this frequency band the magnitude flatness is less than $2.568 \mathrm{~dB}$ after the MFNN's equalization.

\section{ANALYSIS OF SPECTRUM}

The methodology of the bandwidth-expanding has been applied to process PD data measured both in laboratory and the electrical substation. The compensation mentioned in following sections means that data has been processed with methodology addressed previous sections.

\section{A. Laboratory Validation}

A tightly controlled set of laboratory tests were conducted to validate the proposed technique with empirically derived data. The tests used one TEM horn to receive pulses radiated from a second mirrored TEM horn. The experimental system consisted of a pulse generator, a pair of TEM horns and a DSO. The schematic of the system is shown in Fig. 14. The pulse generator is Picosecond's 10060A with features of leading edge transition time $55 \mathrm{ps}$ and pulse duration $10 \mathrm{ps}-$ 10ns. The DSO is LeCroy's SDA 9000. A pair of the TEM horns is either LB horns or HB horns. The pulses from the $10060 \mathrm{~A}$ pulse generator were radiated out through one TEM horn and the mirrored TEM horn captured the pulses. The captured pulses were sampled and stored in memory by the DSO. The sampled data were then processed with the method described in Section III. Specimen results are shown in Fig. $15-18$.

Fig. 15 shows time-domain signals received by the LB horn for a $10 \mathrm{~ns}$ pulse and Fig. 16 shows the frequencydomain representation within frequency range $710 \mathrm{MHz}-$ 2.1GHz. Fig. 17 shows the time-domain signals received by HB horn for a 100 ps pulse and Fig. 18 shows the frequencydomain representation within frequency range 2.1-6 GHz. The --- line represents the original signal while the -line represents the compensated signal in these plots.

By analysis of the results produced, the spectrum of the original pulse (detected by the LB TEM horn) is not observed to be significantly different from that of the compensated pulse. This is because the intended compensation level is reasonably small as shown in Fig.12. However, the amplitude response to frequency magnitude of the compensate pulse is smoother than that of the original pulse detected with HB TEM horn within frequency range $2.1-6 \mathrm{GHz}$. Meanwhile the compensated spectrum magnitude is clearly larger than the original signal's. This is just intended to match the LB TEM horn's magnitude level.

\section{B. PD Measured from a PD simulator}

In order to make LB and HB horns possible simultaneously capture a same PD each time, PD source was an simulator, developed previously by one of authors [19], at the field. The field is the Control Building of $400 \mathrm{kV}$ Strathaven electrical substation. The measurement system is that described in Section II. An example of PD measured by the LB and HB horns is shown in Fig. 19 and 21, respectively.

Figs 19 and 20 show the time domain signal and its frequency domain representation (in the range $710 \mathrm{MHz}-$ $2.1 \mathrm{GHz}$ ) respectively for the LB horn. The -- line represents the spectrum of the measured PD and the - line represents the spectrum of the compensated PD.

Similarly figs 21 and 22 show the time domain signal and frequency representation (in the range $2.1 \mathrm{GHz}-6 \mathrm{GHz}$ ) respectively for the $\mathrm{HB}$ horn. The --- line represents the spectrum of the measured $\mathrm{PD}$ and the - line represents the spectrum of the compensated PD.

By analysis of LB TEM horn's spectrum as shown in Fig. 20 , there are characteristics like that the magnitude of the spectrum from the measured $\mathrm{PD}$ is mainly within range -155$142 \mathrm{~dB} / \mathrm{Hz}$ while that from the compensated PD is mainly within range $-155-140 \mathrm{~dB} / \mathrm{Hz}$. By analysis of HB TEM horn's spectrums as shown in Fig. 22, there are characteristics like that the magnitude of the spectrum from the measured PD is mainly within range $-160-143 \mathrm{~dB} / \mathrm{Hz}$ while that from the compensated $\mathrm{PD}$ is mainly within range $-155-140 \mathrm{~dB} / \mathrm{Hz}$. Comparing LB horn's spectrum with HB horns', there are characteristics like that there are about $5 \mathrm{~dB} / \mathrm{Hz}$ difference for magnitudes of the spectrums before compensation between LB TEM horn and HB TEM horn. However, the magnitudes of the spectrums for LB TEM horn and HB TEM horn are mainly in similar level after compensation.

\section{Measurements in an Electrical Substation}

The detection system developed in this study has been deployed at $400 \mathrm{kV}$ power substation. The measurements carried out 24 hours a day for a prolonged period. The purpose of the measurements was to characterize impulsive noise at electrical substations. A typical time domain trace is shown in Fig. 23 and frequency domain representation in Fig. 24. From analysis of the spectrum, there are ultra wide band (UWB) frequency components from $0.5 \mathrm{MH}$ to $5 \mathrm{GHz}$ present. The most significant frequency components are 0.5 $1.2 \mathrm{MHz}, 90-108 \mathrm{MHz}, 177-285 \mathrm{MHz}, 500 \mathrm{MHz}, 600-$ $750 \mathrm{MHz}, 833 \mathrm{MHz}, 1.25 \mathrm{GHz}, 1.666 \mathrm{GHz}, 1.76 \mathrm{GHz}, 2.1 \mathrm{GHz}$, $2.917 \mathrm{GHz}, 3.333 \mathrm{GHz}, 3.75 \mathrm{GHz}$ and $5 \mathrm{GHz}$. Refer to UK OFCOM's radio frequency allocations [20], some frequency components fall into some specific bands. The $0.5-1.2 \mathrm{MHz}$ are MW AM broadcasting bands. The $90-108 \mathrm{MHz}$ is the FM radio bands. The $177-285 \mathrm{MHz}$ is Public Access Mobile Radio (PAMR), JFMG for programme-making, live entertainment, special events, NATO military and Space Shuttle bands. The $500 \mathrm{MHz}, 600-750 \mathrm{MHz}, 833 \mathrm{MHz}$ are TV broadcasting bands. The $1.25 \mathrm{GHz}$ is civil airport Radar 
band. The $1.76 \mathrm{GHz}$ and $2.1 \mathrm{GHz}$ are GSM 1800 and $3 \mathrm{G}$ bands, respectively. The $2.917 \mathrm{GHz}$ is civil maritime, air traffic control and range safety radars, together with naval radars. The $3.333 \mathrm{GHz}$ is maritime mobile band. The 3.75 $\mathrm{GHz}$ is C-Band satellite TV band. The $5 \mathrm{GHz}$ is Microwave Landing System band.

Although the UWB signals were detected within a substation, the most of them are noises to impulsive noise which is targeted to. The impulsive noise components need to be distilled from the measurements recorded on site. However, this is out of the scope of this paper and may be reported in subsequent publications.

\section{CONCLUSION}

A system for PD detection at an electrical substation has been described. The methodology of bandwidth-expanding technique for the detection system has been reported. The MFNNs for the equalization amplitude response to frequency magnitude has been discussed. The example results processed with the methodology have been presented. The methodology by using the MFNN has made detection SHF PD practically possible, beyond the hardware limits and a breakthrough to conventional way of detection VHF or UHF PD. Due to the detection with the system is UWB signals, it is a challenge to extract real impulsive noise of power substation from measurements. Further work is underway to investigate a methodology of processing measurement signals in the substation. The system developed in this study may be potentially used for conditioning monitoring HV electricity equipment. In this case a portable single TEM horn for SHF PD detection needs to further investigate based on the bandwidth-expand methodology in this study.

\section{ACKNOWLEDGMENT}

The preferred spelling of the word "acknowledgment" in American English is without an "e" after the "g." Use the singular heading even if you have many acknowledgments. Avoid expressions such as "One of us (S.B.A.) would like to thank ... ." Instead, write "F. A. Author thanks ... ."

\section{REFERENCES}

[1] IEEE Standard, "Definition, Specification, and Analysis of Systems Used for Supervisory Control, Data Acquisition, and Automatic Control," in IEEE Standard C37.1, 1994.

[2] IEC Standard, "Communications Networks and Systems in SubstationsPart1: Introduction and overview," in IEC Standard 61850-1, 2003.

[3] R. Ehlers, "Using UCA to integrate and deliver diagnostic data from substation," in 12th substation equipment diagnostics conference $\mathrm{New}$ Orleans, USA, 2004.

[4] H. G. Sedding, S. Bomben, S. Rizzeto, and R. Densley, "Assessment of station cable condition using wireless telemetry and diagnostic tests," in 2001 EEIC conference, 2001

[5] P. Duggan, P. DilLilo, P. DiScala, R. Schwabe, J. Braun, N. Fujimoto, and S. Rizzetto, "Field experience with live wireless telemetry sensors on power equipment operating to $345 \mathrm{kV}$," in 12 th substation equipment diagnostics conference New Orleans, USA, 2004.

[6] "Radio noise," in ITU-R Recommendation, 2003, pp. 372-378.

[7] I. A. Glover, Q. Shan, P. J. Mooore, I. E. Portugues, and R. J. Watson "An investigation into the vulnerability of WLAN and WPAN technologies to impulsive noise in electricity transmission substations," in Ninth International Symposium on Communication Theory and Applications ISCTA'07, Ambleside, Lake District, UK, 2007.

[8] P. J. Moore, I. E. Portugues, and I. A. Glover, "Radiometric location of partial discharge sources on energized high-Voltage plant," Power Delivery, IEEE Transactions on, vol. 20, pp. 2264-2272, 2005.

[9] T. Sakakibara, H. Murase, E. Haginomori, S. Wakabayashi, K. Emoto, and A. Ogawa, "Study of propagation phenomena of partial discharge pulses in gas insulated substation," Power Delivery, IEEE Transactions on, vol. 13, pp. 768-776, 1998.

[10] M. D. Judd, O. Farish, J. S. Pearson, and B. F. Hampton, "Dielectric windows for UHF partial discharge detection," IEEE Transactions on Dielectrics and Electrical Insulation, vol. 8, pp. 953-958, 2001.

[11] T. Huecker and J. Gorablenkov, "UHF partial discharge monitoring and expert system diagnosis," Power Delivery, IEEE Transactions on, vol. 13, pp. 1162-1167, 1998.

[12] R. Kurrer and K. Feser, "The application of ultra-high-frequency partial discharge measurements to gas-insulated substations," Power Delivery, IEEE Transactions on, vol. 13, pp. 777-782, 1998.

[13] P. J. Moore, I. Portugues, and I. A. Glover, "A nonintrusive partial discharge measurement system based on RF technology" in Power Engineering Society General Meeting, 2003, IEEE, 2003, p. 633 Vol. 2.

[14] Q. Shan, I. A. Glover, P. J. Moore, I. E. Portugues, M. Judd, R. Rutherford, and R. J. Watson, "TEM Horn Antenna for Detection of Impulsive Noise," in EMC Europe 2008, Hamburg, Germany, 2008.

[15] I. E. Portugues, P. J. Mooore, and I. A. Glover, "An investigation into the effect of receiver bandwidth for the interpretation of partial discharge impulses using remote radio sensing," in International University Power Engineering Conference, 2002, pp. 529-533.

[16] M. Negnevitsky, "Artificial Neural Network," in Artificial Intelligence London: Pearson Education Limited, 2002.

[17] S. Haykin, "A comprehensive foundation," in Neural Networks New Jersey: Prentice Hall, 1999.

[18] M. T. Hagan and M. B. Menhaj, "Training feedforward networks with the Marquardt algorithm," Neural Networks, IEEE Transactions on, vol. 5, pp. 989-993, 1994.

[19] S. Xiao, P. J. Moore, M. D. Judd, and I. E. Portugues, "An Investigation into Electromagnetic Radiation due to Partial Discharges in High Voltage Equipment" in Power Engineering Society General Meeting, 2007. IEEE, 2007, pp. 1-7.

[20] UK OFCOM, "United Kingdom table of radio frequency allocations $9 \mathrm{kHz}$ - 105GHz," Accessed on 07/07/2008 13:24, Available: http://www.ofcom.org.uk/static/archive/ra/publication/ra info/ra365.ht m.IEEE Criteria for Class IE Electric Systems (Standards style), IEEE Standard 308, 1969

First A. Author (M'76-SM'81-F'87) and the other authors may include biographies at the end of regular papers. Biographies are often not included in conference-related papers. This author became a Member (M) of IEEE in 1976, a Senior Member (SM) in 1981, and a Fellow (F) in 1987. The first paragraph may contain a place and/or date of birth (list place, then date). Next, the author's educational background is listed. The degrees should be listed with type of degree in what field, which institution, city, state, and country, and year degree was earned. The author's major field of study should be lower-cased.

The second paragraph uses the pronoun of the person (he or she) and not the author's last name. It lists military and work experience, including summer and fellowship jobs. Job titles are capitalized. The current job must have a location; previous positions may be listed without one. Information concerning previous publications may be included. Try not to list more than three books or published articles. The format for listing publishers of a book within the biography is: title of book (city, state: publisher name, year) similar to a reference. Current and previous research interests end the paragraph.

The third paragraph begins with the author's title and last name (e.g., Dr. Smith, Prof. Jones, Mr. Kajor, Ms. Hunter). List any memberships in professional societies other than the IEEE. Finally, list any awards and work for IEEE committees and publications. If a photograph is provided, the biography will be indented around it. The photograph is placed at the top left of the biography. Personal hobbies will be deleted from the biography.

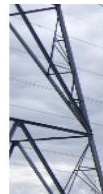




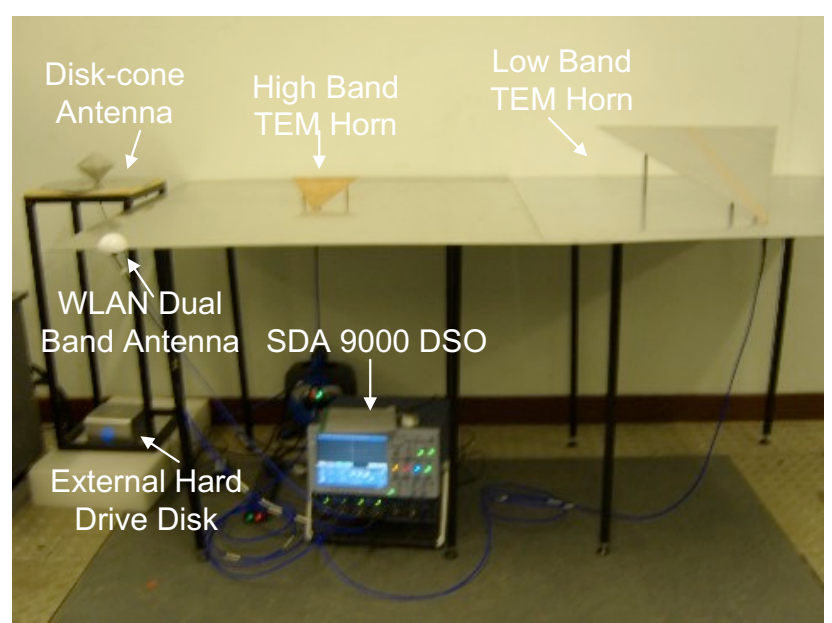

Fig. 2. The PD detection system was deployed in the control room of the $400 \mathrm{kV}$ power network.

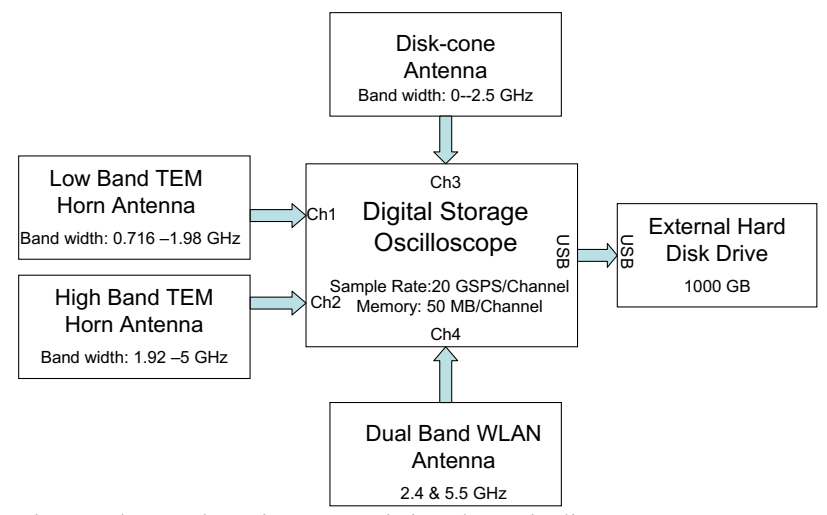

Fig. 3. The PD detection system is in schematic diagram.

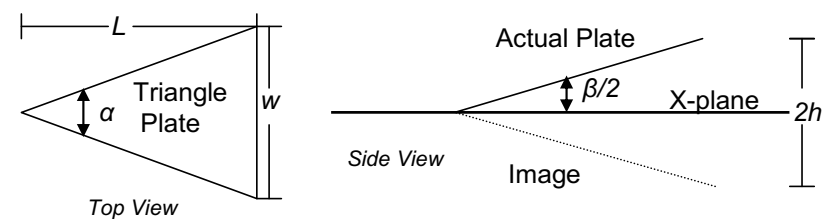

Fig. 4. Schematic diagram of TEM horn antenna.
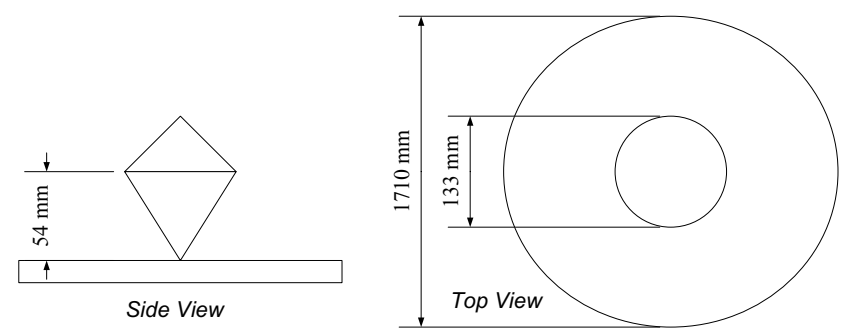

Fig. 5. Schematic diagram of disk-cone antenna.

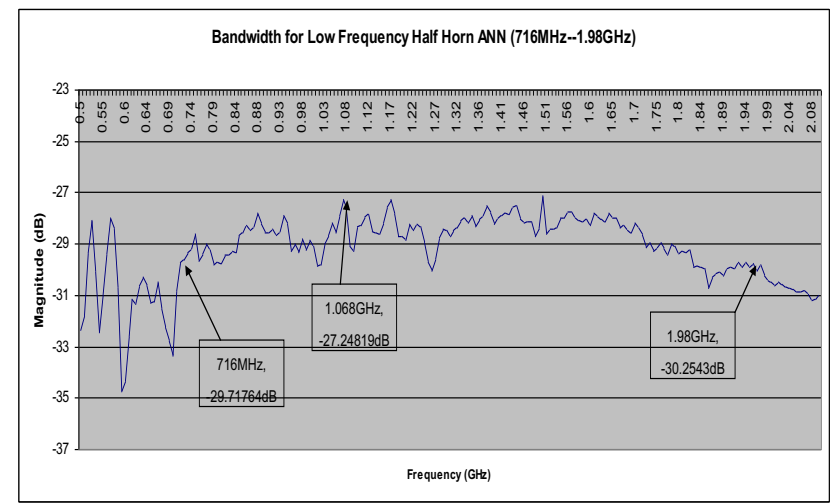

Fig. 6. The LB TEM horn's amplitude response to the frequency magnitude is measured.

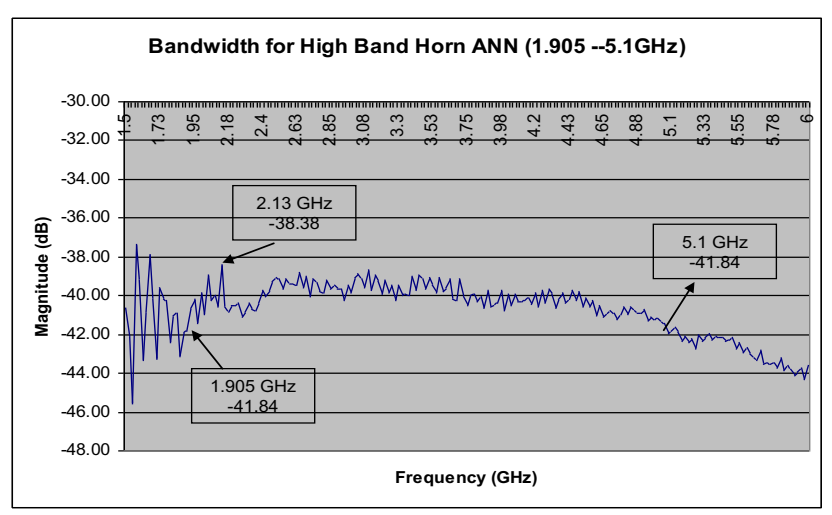

Fig.7. The HB TEM horn's amplitude response to the frequency magnitude is measured.

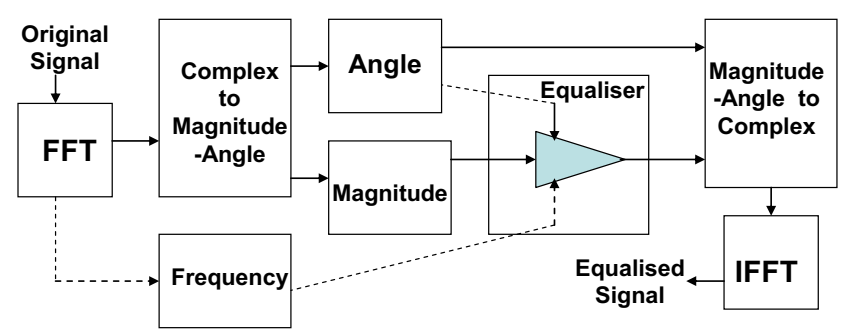

Fig. 8. The frequency-band-expanding methodology.

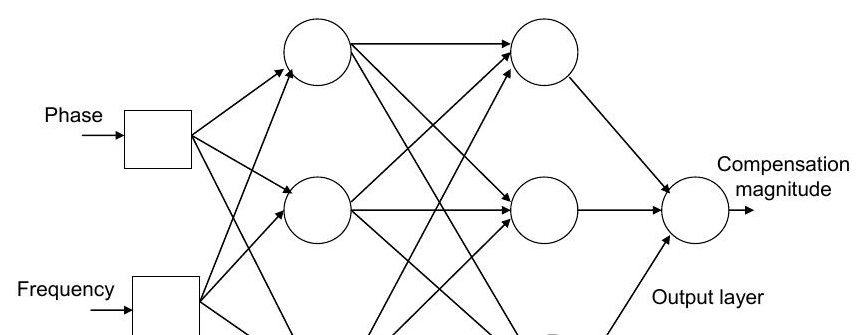




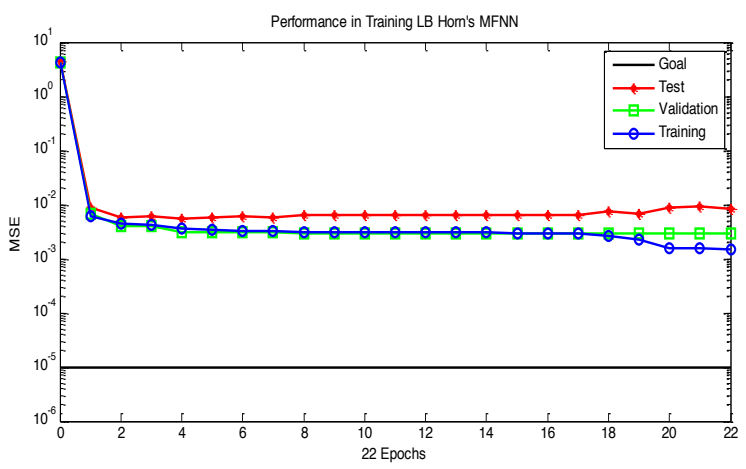

Fig.10. Performance in training LB horn's MFNN.

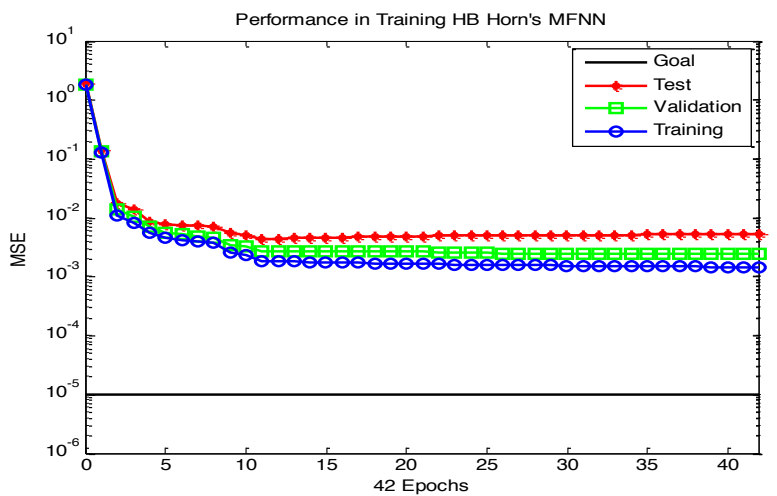

Fig.11. Performance in training HB horn's MFNN.

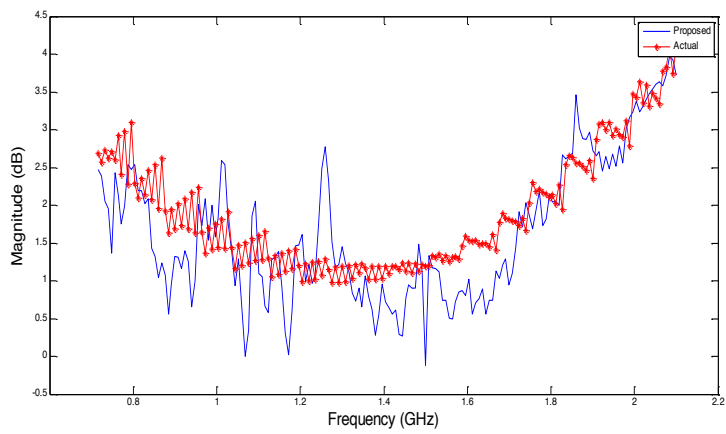

Fig.12. The proposed compensation and actual achieved by MFNN in amplitude response vie frequency magnitude for LB Horn.

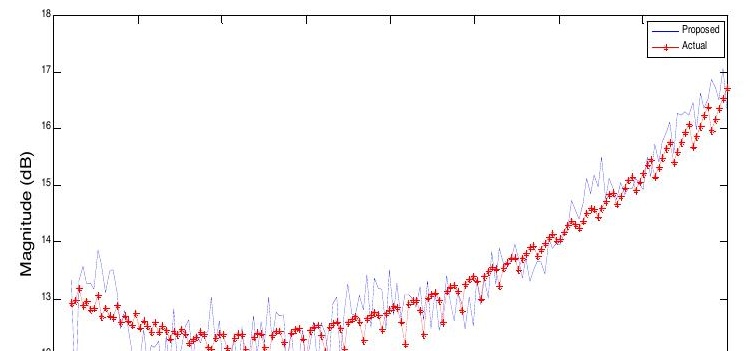

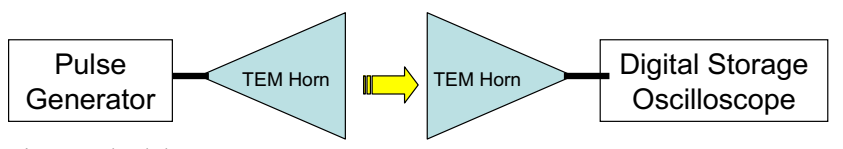

Fig.14. The laboratory test system.

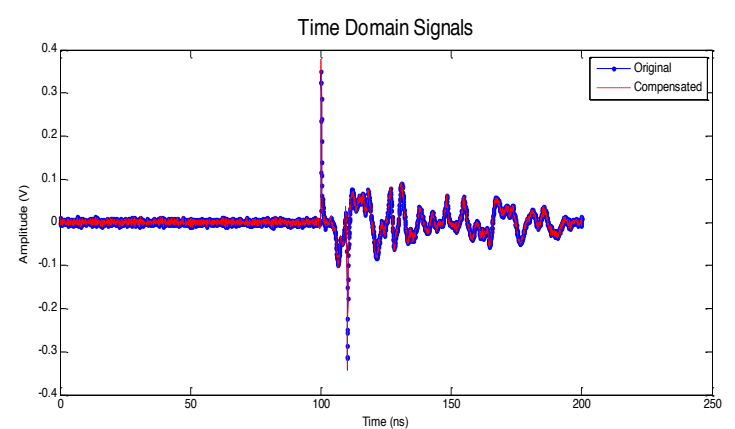

Fig.15. The time-domain original and compensated signals, measured by LB horn.

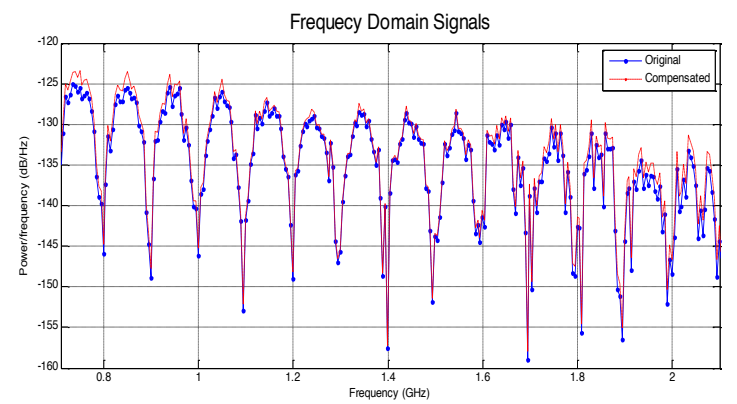

Fig.16. The frequency-domain original and compensated signals, measured by LB horn.

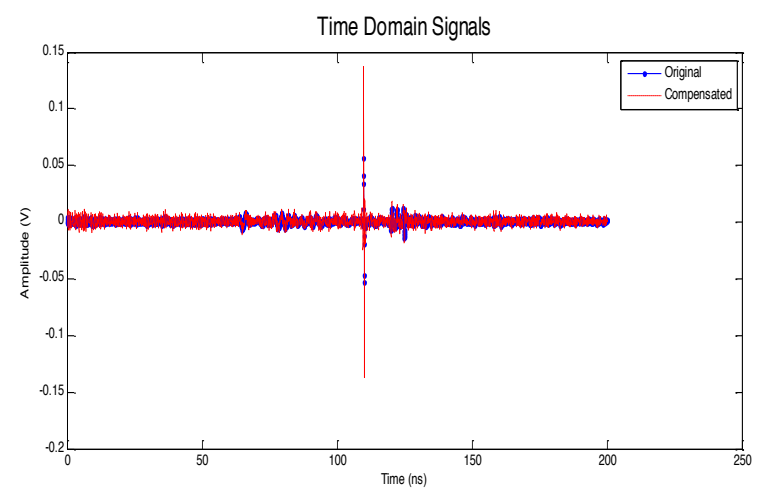

Fig.17. The time-domain original and compensated signals, measured by HB horn. 


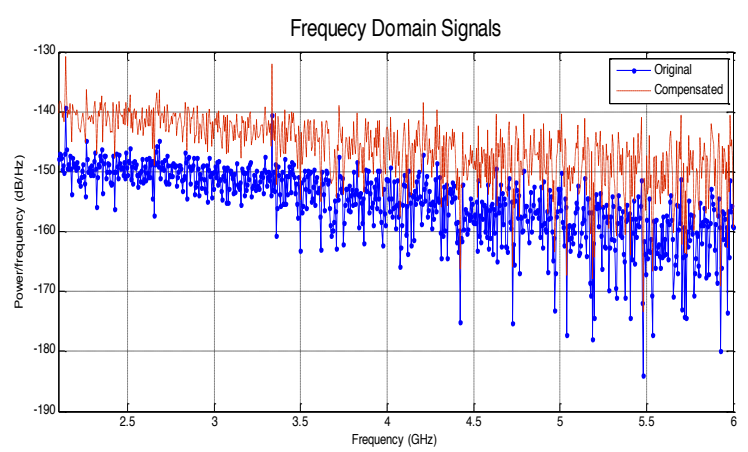

Fig.18. The frequency-domain original and compensated signals, measured by HB horn.

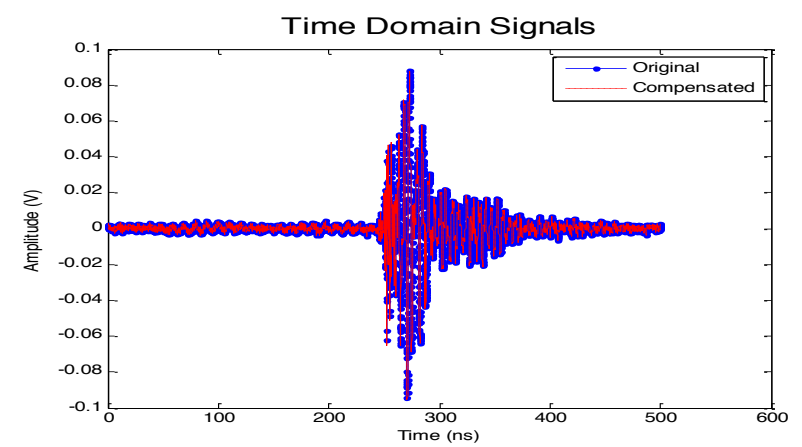

Fig.19. The time-domain original and compensated signals, measured by LB horn at power substation.

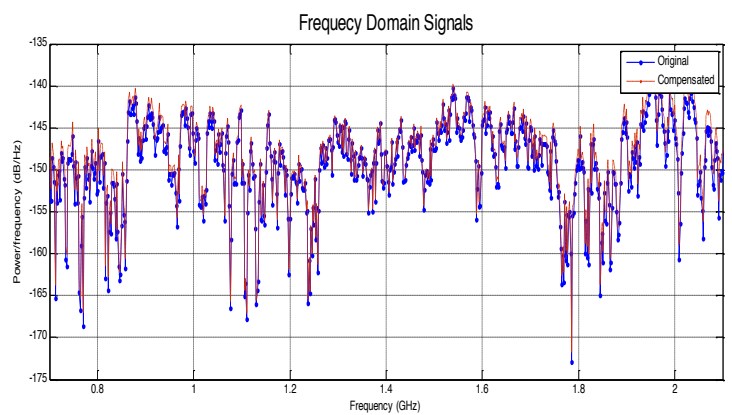

Fig.20. The frequency-domain original and compensated signals, measured by LB horn at power substation.

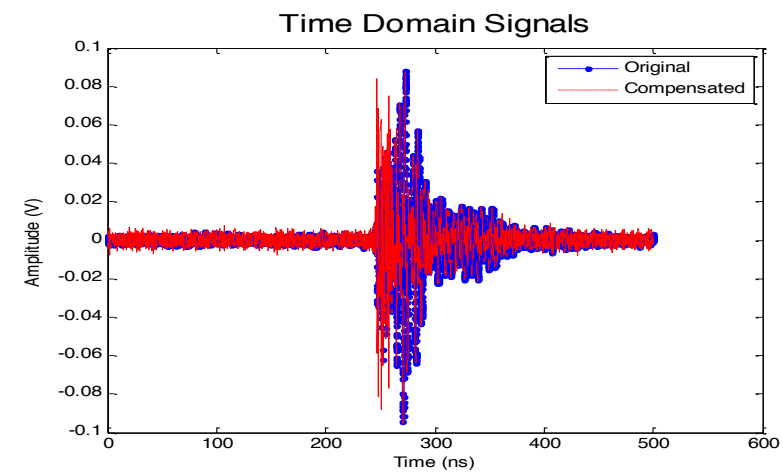

Fig.21. The time-domain original and compensated signals, measured by $\mathrm{HB}$ horn at power substation.

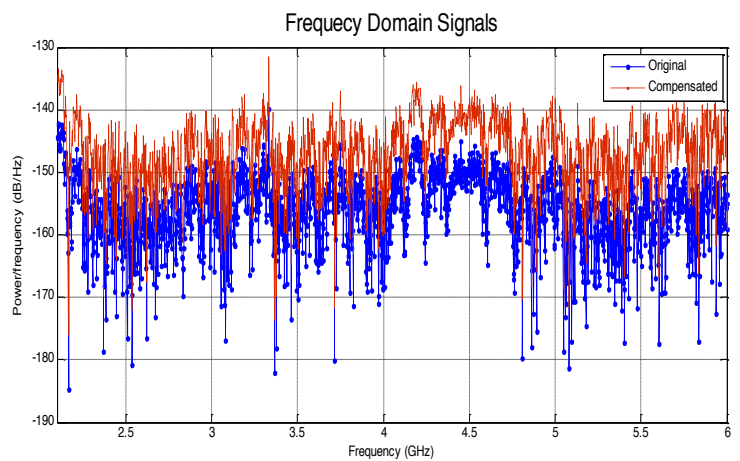

Fig.22. The frequency-domain original and compensated signals, measured by HB horn at power substation.

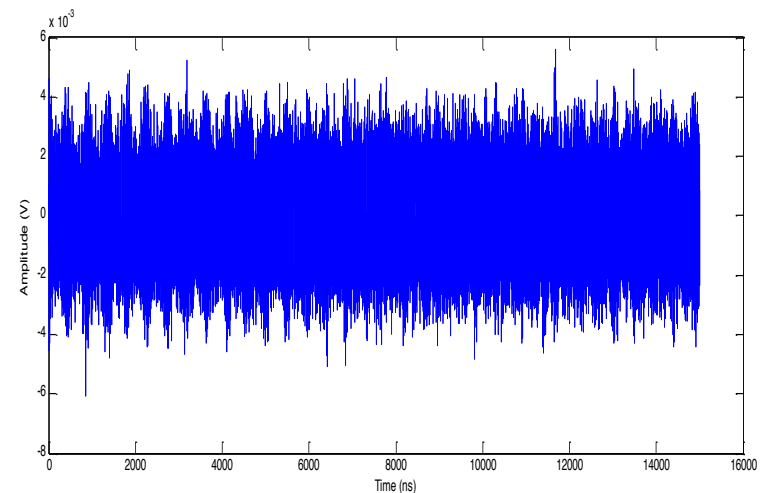

Fig.23. A result measured in $400 \mathrm{kV}$ power substation.

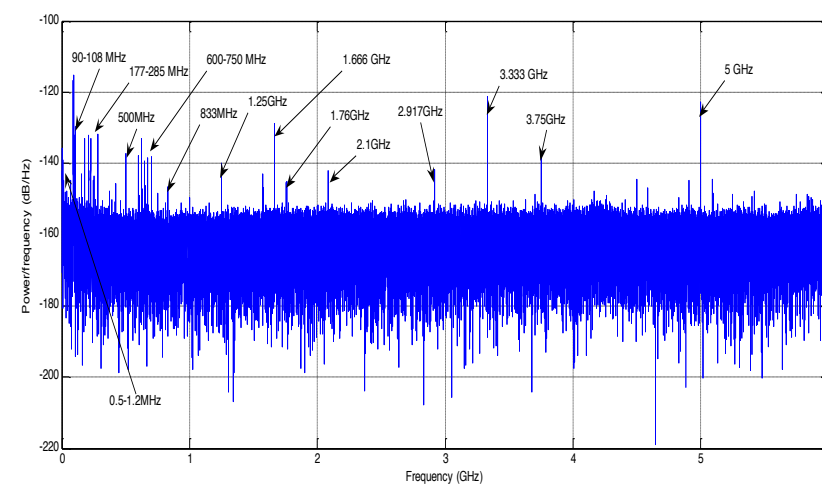

Fig.24. The spectrum of the measurement from $400 \mathrm{kV}$ power substation. 
